\title{
Utilidad del ultrasonido intracoronario en el diagnóstico de disección coronaria espontánea
}

\author{
Utility of intravascular ultrasound in the diagnosis of spontaneous coronary dissection
}

Nelson Amores y Paúl Salvador*

Departamento de Cardiología, Hospital Carlos Andrade Marín, Quito, Ecuador

\section{Presentación de dos casos}

Mujer de 32 años, sin factores de riesgo cardiovascular; niega uso de anticonceptivos hormonales. Acude a nuestro Servicio por ángor al reposo de aproximadamente siete horas de evolución; el electrocardiograma inicial muestra un infarto con ST elevado anterior extenso. Se le realizó una coronariografía, en la cual se observó una lesión angiográficamente severa en descendente anterior (DA) proximal. En la evaluación con ultrasonido intracoronario (IVUS) se objetivó una zona de disección desde origen a segmento medio de la DA (Fig. 1). El ecocardiograma transtorácico evidenció acinesia anteroseptoapical con función ventricular preservada (59\% Simpson biplano). Se decidió no realizar ninguna intervención coronaria y vigilar, con optimización del tratamiento farmacológico de manera conservadora. La evolución fue satisfactoria al primer y tercer mes de seguimiento, sin presentar ángor recurrente o disminución en su clase funcional. Recibe ácido acetiIsalicílico (AAS), calcioantagonista, betabloqueador y nitrato.

Mujer de 40 años de edad, sin factores de riesgo cardiovascular; niega uso de anticonceptivos hormonales. Acude al Servicio de emergencia por angina típica en reposo que se presentó en la madrugada mientras dormía. En el momento de la evaluación está asintomática. El electrocardiograma inicial muestra lesión subepicárdica en cara anterior; mínima movilización enzimática. Fue catalogada como angina inestable y se le realizó una coronariografía que identificó una lesión no significativa en el tercio medio de la DA. Con la ayuda de IVUS se objetivó una zona de disección en tercio medio de la DA (Fig. 2). Se decidió no realizar ningún intervencionismo coronario. El ecocardiograma evidenció función ventricular preservada (66\% Simpson biplano), sin trastornos de motilidad segmentaria. Evolución satisfactoria, recibe AAS, inhibidor de la enzima convertidora de angiotensina y betabloqueador, se encuentra libre de ángor u otros síntomas con un seguimiento de cuatro meses.

La disección coronaria se caracteriza por la rotura de la capa íntima coronaria, que permite el flujo de sangre hacia una falsa luz en la capa media, lo que oblitera la luz verdadera y provoca insuficiencia coronaria $^{1-3}$.

Se ha observado una prevalencia mucho más alta en series de reporte de casos contemporáneas, es causa de hasta el $25 \%$ de todos los casos de síndrome coronario agudo en mujeres $<50$ años de edad. Sin embargo, se considera que aún existe infradiagnóstico, esto debido a su bajo perfil de riesgo en series

\section{Correspondencia:}

*Paúl Salvador

Servicio de Cardiología

Hospital Carlos Andrade Marín

Av. Universitaria y Ayacucho, N19-63

Fecha de recepción: 18-11-2017

Fecha de aceptación: 03-08-2018

E-mail: paulsalvador90@ hotmail.com DOI: 10.24875/ACM.M19000009
Disponible en internet: 19-03-2019 Arch Cardiol Mex. 2019;89(1):82-84 www.archivoscardiologia.com

1405-9940 @ 2019 Instituto Nacional de Cardiología Ignacio Chávez. Publicado por Permanyer México SA de CV. Este es un artículo Open Access bajo la licencia CC BY-NC-ND (http://creativecommons.org/licenses/by-nc-nd/4.0/). 


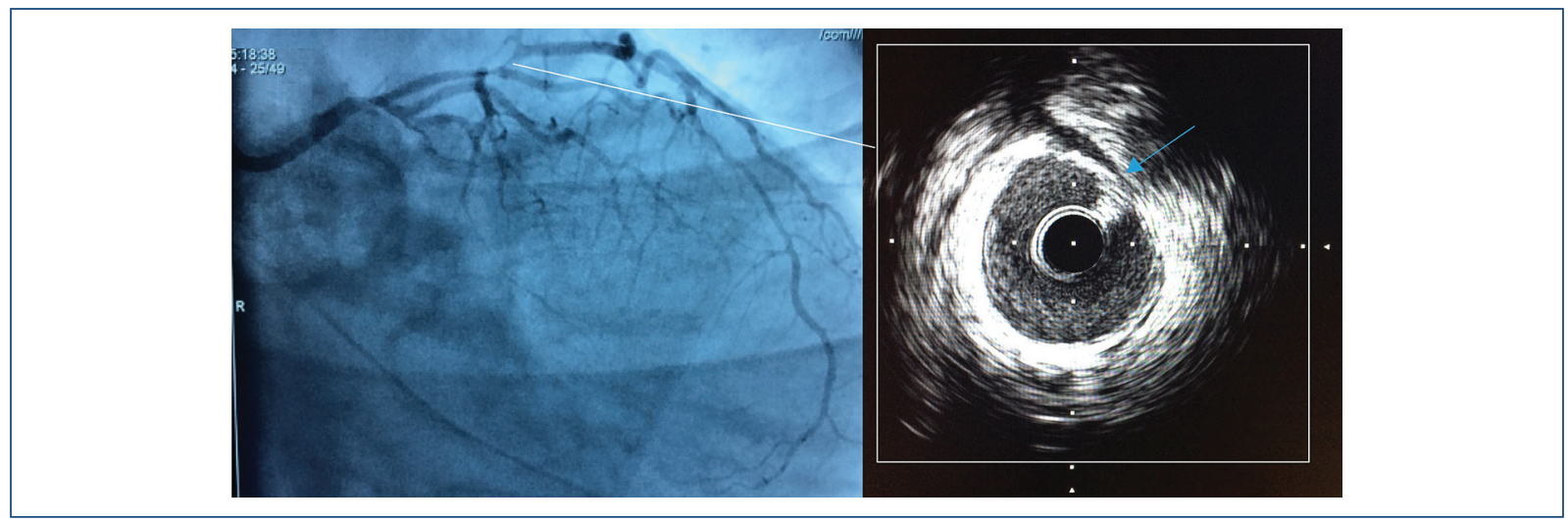

Figura 1. Coronariografía y ultrasonido intracoronario correspondiente a sitio de disección (coronaria descendente anterior segmento proximal) con presencia de flap de disección (flecha) y hematoma intramural.

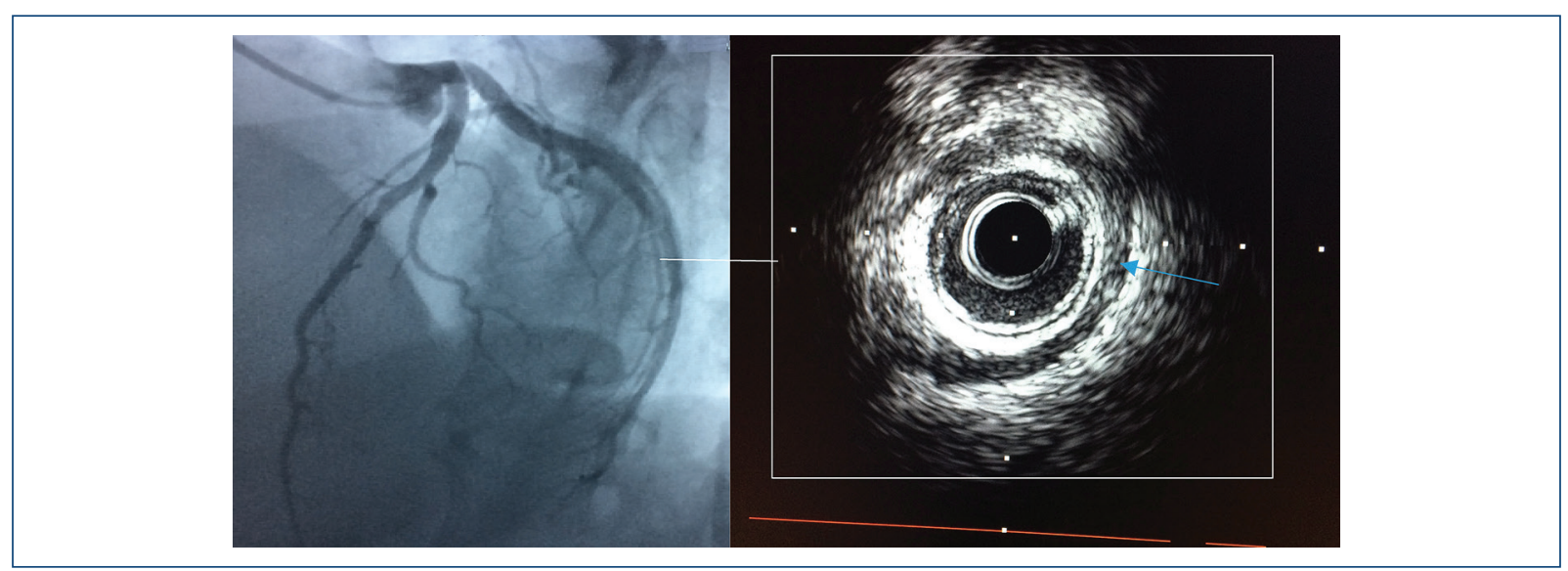

Figura 2. Coronariografía y ultrasonido intracoronario correspondiente a sitio de disección (coronaria descendente anterior segmento medio a distal) con presencia de hematoma intramural (segmento medio) (flecha).

poblacionales. El desarrollo y masificación de técnicas de imagen intracoronaria permiten una mayor identificación de esta enfermedad, con un aumento progresivo en su incidencia ${ }^{3}$.

Afecta con más frecuencia al sexo femenino, las series publicadas muestran una relación 3:1. Otros factores de riesgo son el periodo gestacional o puerperal $^{4}$, la presencia de aterosclerosis coronaria, la terapia estrogénica, la coexistencia de enfermedades sistémicas, la migraña, las enfermedades del tejido conectivo y, más recientemente, se ha determinado una relación más clara con la fibrodisplasia muscular ${ }^{3,4}$.

La presentación más frecuente de la enfermedad es como un infarto con elevación del segmento ST, que involucra la cara anterior del ventrículo izquierdo, pues el compromiso de la arteria descendente anterior es mucho más frecuente que la de otras arterias ${ }^{4,5}$.
La angiografía coronaria invasiva permite realizar el diagnóstico mediante tres tipos de presentación angiográfica: 1) evidencia de extravasación de contraste creando doble lumen; 2) estrechamiento de la luz superior a $20 \mathrm{~mm}$ de longitud, sin cambio con la administración de nitroglicerina y sin evidencia de enfermedad aterosclerótica en otras arterias, y 3) estenosis focal similar a una lesión aterosclerótica que precisa de imagen intracoronaria para su confirmación $n^{4}$.

La utilización del IVUS y la tomografía de coherencia óptica permiten caracterizar con fino detalle la lesión, confirmando el diagnóstico y estimando la extensión de la disección. El hallazgo de un flap de disección intimomedial y de un hematoma intramural son los criterios utilizados ${ }^{5,6}$.

Debido a la baja incidencia e infradiagnóstico de esta patología no existen suficientes estudios clínicos aleatorizados con número adecuado de pacientes que 
ayuden a tomar una decisión basada en evidencia, por eso el análisis resolutivo será paciente a paciente, basado en: condición clínica inicial, riesgo de sangrado, respuesta o no al tratamiento conservador, anatomía de la lesión y compromiso o no de varias arterias?

Diversos estudios observacionales y un reciente metaanálisis han demostrado que el tratamiento conservador se elige como primera opción, pues ha demostrado mejores resultados intrahospitalarios. La intervención coronaria percutánea representa un reto en estos casos debido a la dificultad técnica de canalizar la luz verdadera del vaso, la cuantificación sesgada del diámetro de la arteria y la friabilidad de la pared arterial al momento de implantar un stent; todo esto provoca un riesgo incrementado de complicaciones agudas y a largo plazo ${ }^{8,9}$.

Se han descrito diferentes estrategias, que incluyen el implante de stents reabsorbibles ${ }^{10}$ y el uso de balones cortantes que realizan fenestraciones en la íntima del vaso y así aliviar la compresión, sin embargo, se precisan mayor cantidad y seguimiento de estos pacientes. En todo caso, el desarrollo de todas estas técnicas ha sido colaborado por el uso de imagen intracoronaria, ya sea ecográfica o por tomografía 6 .

En relación con la revascularización quirúrgica, esta ha sido relegada como última opción terapéutica debido al riesgo que conlleva anastomosar un puente a la luz falsa y la alta incidencia de reoclusiones. Esta opción es considerada cuando el intervencionismo coronario falla y existe buen lecho distal ${ }^{9}$.

En relación con el tratamiento conservador, la terapia antiagregante y anticoagulante como estrategia inicial en el contexto de una presentación de síndrome coronario agudo es discutible luego del diagnóstico de disección coronaria espontánea. Evidentemente, el uso de antiagregantes está indicado cuando se tomó la indicación de colocar un stent, y por otro lado su uso como prevención de recurrencias sin intervencionismo es incierto. El uso de anticoagulación supone un riesgo al estar relacionado como favorecedor de aumento de disección, así que su indicación debe relegarse a casos en los cuales el riesgo embólico sea elevado como: tromboembolia o trombo mural en el ventrículo izquierdo. El uso de estatinas es muy discutido, debido a que generalmente estos pacientes no tienen componente aterotrombótico y además ha demostrado aumento de recurrencias de disección coronaria espontánea, según un estudio observacional ${ }^{3,5,9}$.

Diversos autores recomiendan uso de betabloqueantes como estrategia de tratamiento, arguyendo que su uso, al disminuir el estrés parietal, contribuiría a disminuir la línea de disección; sin embargo, esta recomendación no ha mostrado disminución en la recurrencia de disección coronaria espontánea ${ }^{10}$.

\section{Conflicto de intereses}

Los autores declaran no tener conflicto de intereses ni haber recibido financiación.

\section{Responsabilidades éticas}

\section{Protección de personas y animales}

Los autores declaran que para esta investigación no se han realizado experimentos en seres humanos ni en animales.

\section{Confidencialidad de los datos}

Los autores declaran que han seguido los protocolos de su centro de trabajo sobre la publicación de datos de pacientes.

\section{Derecho a la privacidad y consentimiento informado}

Los autores declaran que en este artículo no aparecen datos de pacientes.

\section{Agradecimientos}

A todo el personal del Servicio de Cardiología por su inestimable apoyo en el día a día de nuestras labores.

\section{Bibliografía}

1. Saw J, Mancini J, Humphries K. Contemporary review on spontaneous coronary artery dissection. J Am Coll Cardiol. 2016;68(14):297-312.

2. Meng PN, Xu C, You W, Wu ZM, Xie DJ, Zhang H, et al. Spontaneous coronary artery dissection as a cause of acute myocardial infarction in young female population: A single-center study. Chin Med J (Engl). 2017;130(13):1534.

3. Tweet M, Kok S, Hayes S. Spontaneous coronary artery dissection in women: What is known and what is yet to be understood. Clin Cardiol. 2018:41(2):1-3.

4. Sánchez Rodríguez AC, Domínguez Rivas MJ, González Alemán O Beíitez Muñoz L, Álvarez-Osorio Cisneros S, Rodríguez Yañez JC. Disección coronaria espontánea. Causa alternativa de isquemia miocárdica en mujeres. Rev. argent. ter. intensiva. 2018:35(1):1-2

5. Saw J, Aymong E, Sedlak T, Buller CE, Starovoytov A, Ricci D, et al. Spontaneous coronary artery dissection association with predisposing arteriopathies and precipitating stressors and cardiovascular outcomes. Circ Cardiovasc Interv. 2014;7(5):645-55.

6. Hun Jang J, Hyeok Kim D, Hyuk Yang D, II Woo S, Kwan J, Soo Park K, et al. Spontaneous coronary artery dissection by intravascular ultrasound in a patient with myocardial infarction. Korean J Intern Med. 2014;29:106-10.

7. Bastante T, Cuesta J, García-Guimaraes M, Rivero F, Maruri R, Adlan D, et al. Current management of spontaneous coronary artery dissection. Expert Rev Cardiovasc Ther. 2017;15(8):619-28.

8. Tweet M, Eleid MF, Best PJM, Lennon RJ, Lerman A, Rihal CS, et al. Spontaneous coronary artery dissection: Revascularization versus conservative therapy. Circ Cardiovasc Interv. 2014;7(6):777-86.

9. Martins JL, Afreixo V, Santos L, Costa M, Santos J, Gonçalves L. Medical treatment or revascularisation as the best approach for spontaneous coronary artery dissection: A systematic review and meta-analysis. Eur Hear J Acute Cardiovasc Care. 2018;7(7):614-23.

10. Mahmood MM, Austin D. IVUS and OCT guided primary percutaneous coronary intervention for spontaneous coronary artery dissection with bioresorbable vascular scaffolds. Cardiovasc Revascularization Med. 2017;18(1):53-7 